\title{
ANALYSE DE LA LEGITIMATION \\ DE LA TRANSITION ET DE LA \\ NOUVELLE RÉPUBLIQUE \\ DÉMOCRATIQUE DU CONGO
}

Christophe LUTUNDULA APALA Pen'APALA ${ }^{1}$

932, Avenue Okito, MBINZA/Pigeon, Ngaliema

Kinshasa

République Démocratique du Congo

CHAMP D'INVESTIGATION: droit constitutionnel

SUMMARY: ANALYSIS OF THE LEGITIMATING OF THE TRANSITION AND OF THE DEMOCRATIC REPUBLIC OF THE CONGO

The aim of this article is to assess the institutional order and the legitimating of the A.F.D.L. government in order to propose some political adjustments which could consolidate the current transition process.

To evaluate the institutional order of the present Congolese Republic, it is necessary to examine the A.F.D.L. Declaration of Assumption of Power and the Constitutional Decree Law number 3. The Declaration of Assumption of Power has important

\footnotetext{
${ }^{1}$ L'auteur était 2ème Vice-Président du Haut Conseil de la République - Parlement de Transition du Zaïre (juin 1994 - avril 1997).
} 
political as well as legal consequences. Politically it stipulates that only the A.F.D.L. is entitled to govern the Congolese state. Legally the Declaration creates a dangerous vacuum. It merely installs a president and a government, and it suspends all former constitutional laws.

The Constitutional Decree Law number 3 was proclaimed to restore the constitutional order and to organise the execution of power. The institutional order established by this Decree Law has two major consequences. It legitimates the otherwise 'unlawful' appointment of some members of the new government, and it establishes a very strong presidential regime without constitutional separation of powers.

The political legitimation of the A.F.D.L. government implies the existence of popular support and international recognition of that government. A strong ground for the overwhelming popular support for the A.F.D.L. is based on Congolese history, especially on the political legacy of Patrice Lumumba, although it has to be said that the legitimating of the present regime is also based on the inadequacy of all former regimes. In addition, it should be noted that the National Sovereign Conference also derived its legitimation from its task to end the existing chaos. Nevertheless, however strong that legitimating may be, now that the old regime is overthrown, chaos still exists and will provoke socio-economic claims. That is the reason why legitimation has to be based on new goals which exceed a simple change of rulers.

Four conclusions can be drawn from this analysis. First of all, the Decree Law is regarded as a very temporary low and consequently it has only established a few pieces of an institutional order. This makes it necessary to create a real republican constitution. Secondly, the existing institutional order risks slipping away into a strong presidential regime. Another consequence is that the present institutional order is open to dictatorship, although it is unlikely that such an evolution can be consolidated by way of social consensus. The challenge will consist in organising a system of political pluralism which can develop into an efficient instrument of development. In the end, good governance implies the social and economic wellbeing as well as the political development of a nation. Finally, the legitimation of the A.F.D.L. is fragile. Therefore it has to be based on a political program which makes national reconciliation possible. Such a reconciliation should include a consensus between the A.F.D.L. and the National Sovereign Conference on the options for a new social contract. 
KEY WORDS : Congo, constitution, Kabila, politics

\section{INTRODUCTION}

La prise de la ville de Kinshasa, le 17 mai 1997, par l'Armée de l'Alliance des Forces Démocratiques pour la Libération du Congo, AFDL, a marqué la fin du régime du Président Mobutu qui a gouverné le Zaïre, aujourd'hui République Démocratique du Congo, pendant 32 ans, sans partage.

Par sa déclaration faite à Lubumbashi à la même date, l'AFDL a pris le pouvoir d'Etat, s'est chargée d'assumer l'autorité de la Transition et a confié la direction du pays à Monsieur Laurent-Désiré Kabila, devenu depuis lors Président de la République, ainsi qu'au gouvernement qu'il a formé peu après. La Transition issue de la Conférence Nationale Souveraine, CNS, prenait fin, par la même occasion, après 5 ans des balbutiements et des ratés interminables.

Par la suite, la classe politique et une bonne partie de la population congolaise, tout en applaudissant le changement intervenu dans la direction du pays, n'ont cessé d'élever, sous diverses formes, des protestations contre la manière dont le nouveau pouvoir est organisé et exercé.

En effet, d'aucuns désapprouvent le rejet en bloc de la CNS par l'AFDL et exigent le respect notamment de l'option démocratique pluraliste qu'elle a levée, tandis que plusieurs réclament l'ouverture aux autres forces politiques et, plus particulièrement, à l'opposition interne non armée qui n'a pas moins combattu la dictature de Mobutu.

D'autres encore mettent en cause, à tort ou à raison, l'opportunité, la régularité, l'impartialité, l'objectivité et l'efficacité de certaines mesures et méthodes de gestion de l'AFDL (interdiction des activités politiques et des manifestations publiques, interpellations, arrestations et détentions des citoyens, réquisitions, 
saisies et confiscations des biens etc...).

Pour leur part, le gouvernement et l'AFDL justifient leur sévérité et les mesures qui sont prises par la nécessité, d'une part, d'assainir les moeurs publiques polluées par une classe politique médiocre et, d'autre part, de redémarrer sur des bases aussi nouvelles que solides. L'agitation actuelle ne serait que les tentatives des Mobutistes et des nostalgiques de l'ancien régime pour restaurer la IIème République honnie par le Peuple congolais.

Si jusque là la tension n'est pas aussi vive que l'on pouvait le craindre, il ne reste pas moins vrai que cette fronde érode le consensus quasi unanime qui s'est créé autour de l'AFDL. Elle pose également le problème de l'adéquation des institutions actuelles aux exigences du changement, de la justification du pouvoir en place et de sa communion avec un Peuple congolais meurtri par 32 ans de dictature implacable.

C'est pourquoi, il est tout autant opportun que nécessaire de développer la réflexion notamment sur le cadre institutionnel et la légitimité du nouveau régime de manière à suggérer, le cas échéant, quelques alternatives pour un ajustement politique susceptible de consolider le processus de changement en cours.

\section{CHAPITRE I : CADRE INSTITUTIONNEL ETABLI PAR L'AFDL}

Pour être pertinente, l'analyse du cadre ou de l'ordre institutionnel de la nouvelle transition ne peut se limiter à décrire l'ordonnancement des principales institutions de la République telles qu'elles sont définies par les textes juridiques. Il importe, comme le suggère Maurice Duverger ${ }^{2}$ d'étudier également le fonctionnement concret de ces institutions pour découvrir la réalité du régime politique actuel au Congo-Kinshasa.

En effet, affirment P.Ch. Goossens et $C$. Zwetkoff, on reconnait volontiers de nos jours qu'une étude juridique des institutions politiques modernes est insuffisante et non pertinente. Elle est impuissante à faire connaître le fonctionnement pratique des régimes politiques. Elle ne saisit les problèmes que sous leur aspect superficiel. La formule de droit, qui est par nature statique, entre nécessairement

${ }^{2}$ DUVERGER, Maurice, Institutions politiques et droit constitutionnel, Paris, Thémis, PUF, Tome 1, 18e édition, 1990 , p. 13. 
en conflit avec le dynamisme politique, et on peut dire que le fonctionnement des institutions politiques dépasse toujours la lettre des constitutions ${ }^{3}$.

Dès lors, le simple commentaire des textes constitutionnels, abstraction faite du contexte socio-politique où ils s'insèrent normalement, aboutit à une distorsion entre le droit et les faits, entre les dispositions constitutionnelles et le fonctionnement réel du pouvoir.

Ainsi, pour une meilleure compréhension de la vie et des institutions politiques d'un pays, il importe d'adjoindre à la méthode juridique l'approche sociologique.

Cette perspective présente l'avantage de tenir compte des données réelles de la vie politique dans l'étude des institutions, de dévoiler les objectifs de celles-ci, d'éclairer leurs mécanismes et de mesurer leur efficacité. ${ }^{4}$

L'examen de la Déclaration de prise de pouvoir par l'AFDL et du Décret-loi constitutionnel $n^{\circ} 003$ du 27 mai 1997 sur lesquels repose l'ordre institutionnel en vigueur en République Démocratique du Congo, procédera en conséquence par cette double dimension juridique et sociologique.

\section{SECTION 1 : DECLARATION DE PRISE DE POUVOIR DU 17 MAI 1997}

Ayant gagné la guerre, il ne restait plus à l'AFDL qu'à prendre le gouvernail du Bateau Zaïre abandonné par son capitaine, le Maréchal Mobutu qui venait, le vendredi 16 mai 1997, de déserter Kinshasa, siège des Institutions de la République, pour Lomé au Togo via Gbadolite.

C'est pourquoi, fort de la situation militaire très favorable sur le terrain et prenant prétexte de la vacance à la tête de l'Etat créée par la fuite du Président Mobutu, le Conseil Elargi de l'AFDL, à l'issue de sa séance extraordinaire de la veille, rendait publique à Lubumbashi le 17 mai 1997 une déclaration dans laquelle il décidait notamment de:

1. Prendre le pouvoir d'Etat et d'assumer désormais l'autorité de transition;

${ }^{3}$ GOOSSENS, P.CH et ZWETKOFF,C. Notes inédites de cours des Institutions politiques comparées, professeur à l'Université de Liège, s.d., pp. 2-3.

${ }^{4}$ LUTUNDULA APALA Christophe, Exécutif du type présidentiel: évolution politique et constitutionnelle au Zaïre, mémoire de Licence, UNAZA, Faculté de Droit 1976-1977, p. 10. 
2. Désigner son Président, Monsieur Laurent-Désiré Kabila, Chef de l'Etat de la République Démocratique du Congo;

3. Constituer un gouvernement de transition de salut public dans un délai de 72 heures;

4. Convoquer une assemblée constituante dans un délai de 60 jours, en vue d'élaborer une Constitution provisoire devant régir la période de Transition;

5. Suspendre tous les actes constitutionnels existants ainsi que les Institutions qu'ils organisent.

A l'évidence, une telle déclaration était lourde des conséquences politiques et juridiques.

Politiquement, en effet, l'AFDL chambardait profondément le paysage de l'ex-Zaïre dont elle devenait l'épicentre. Cette démarche ne pouvait étonner quand on se rappelle le radicalisme exprimé à maintes reprises par ses responsables dans leurs multiples déclarations. Ceux-ci ont toujours soutenu que l'Alliance était la seule force politique véritablement opposée au Président Mobutu; les autres, en ce compris l'opposition non armée, n'étant que Mobutistes. Par conséquent, il n'est nullement question de composer ou de partager avec elles quoi que ce soit. C'est à l'AFDL seule que revient le droit de commander la Nation, de gérer l'Etat et d'occuper l'espace politique congolais, du moins jusqu'aux élections.

La lutte armée et la victoire militaire qu'elle venait de remporter, l'ont confortée dans cette thèse.

Au demeurant, aux négociations de Lomé, de Pretoria et de Pointe-Noire à bord du navire sud-africain, l'Outenica, l'Alliance n'a cessé de revendiquer la remise-reprise avec le Président Mobutu. Est-ce peut-être là aussi une des raisons qui expliquent le rejet catégorique de la Conférence Nationale Souveraine par le Président Kabila et l'AFDL?

En tous cas, la vision de la transition par l'Alliance est manifestement en contradiction avec les principes de la neutralité et du partage équitable et équilibré du pouvoir préconisés par la CNS et les négociations politiques de 1993 dites du Palais du Peuple 
Par ailleurs, se définissant comme un mouvement de rassemblement de toutes les forces vives de la Nation congolaise, il est permis de penser que, si l'AFDL ne se confond pas avec cette Nation, elle en a eu moins l'ambition. Ses hymne, emblème et patrimoine ne s'identifient-ils pas à ceux de la Nation et de l'Etat en vertu des articles 17 et 23 de ses statuts? ? $^{6}$ A coup sur, pareille vision favorise très peu le développement des autres formations politiques qui sont acculées à gagner les rangs du rassemblement lorsqu'elles ne se dissolvent pas tout simplement. Il est fort possible que la suspension des activités des partis politiques et des manifestations publiques, en réalité de celles organisées par d'autres formations que l'AFDL, procède de cette stratégie de neutralisation à petit feu.

Vainqueur de Mobutu, c'est tout naturellement que l'AFDL, à l'instar d'un parti victorieux aux élections, confiera la plus haute fonction de l'Etat à son Président et décidera d'assumer seule l'autorité de transition, c'est-à-dire d'exercer le pouvoir et de gouverner sans partage pendant cette période.

Les déclarations du Secrétaire Général Adjoint de l'Alliance selon lesquelles l'entrée au Gouvernement des personnalités issues des autres partis politiques valait ou impliquait leur adhésion à l'Alliance, n'ont fait que confirmer cette position.

De même, la suspension puis la dissolution du Haut Conseil de la République-Parlement de Transition au sein duquel l'Alliance ne se reconnaissait pas du tout, n'a été que la suite logique d'une démarche qui visait à prendre le contre-pied de la possibilité de poursuivre la transition avec la participation de l'AFDL selon le schéma de la CNS et des négociations du Palais du Peuple, envisagée par les autorités de Kinshasa avant la chute de la Capitale.

Tous comptes faits, la déclaration du 17 mai 1997 n'a laissé l'initiative politique qu'à l'AFDL qu'elle a par ailleurs- érigée en unique source du pouvoir.

Bien plus, elle n'a prévu que deux Institutions de la République: le Président et le Gouvernement. Seulement, si politiquement quelques cases étaient remplies de cette façon, au plan juridique cette déclaration a créé un vide total qui pouvait s'avérer dangereux dans la conduite du pays.

En suspendant tous les textes constitutionnels, elle transformait le Congo en Etat de fait. En outre, privés de matrice constitutionnelle, les lois et règlements en

${ }_{6}^{5}$ Statuts de l'AFDL publiés par le journal le Soft du 30 mai 1997, pages 5 et 6.

${ }^{6}$ Ibidem. 
vigueur tant au pénal qu'au civil étaient, sinon abrogés, à tout le moins suspendus. L'action du pouvoir judiciaire ne pouvait, en conséquence, que s' arrêter.

On s'est demandé à bon droit sur quelle base et par quel acte le nouveau Président de la République allait former le gouvernement. L'acuité de la question est devenue d'autant plus évidente que les premiers membres du gouvernement ont été nommés par un acte législatif; en l'espèce un décret-loi, et sur pied des statuts de l'AFDL fait priver, qui, normalement, ne peut engager tous les citoyens. Dans l'entre-temps, le Président de la République se préparait à prêter le serment constitutionnel à la Nation.

Dans ce contexte, le Décret-loi constitutionnel $n^{\circ} 003$ du 27 mai 1997 tombait à pic. Car il était devenu impérieux et urgent de tracer un cadre juridique minimal, en attendant l'élaboration de la nouvelle constitution de la transition par l'Assemblée Constituante qui devait être convoquée dans 60 jours.

\title{
SECTION 2 : DECRET-LOI CONSTITUTIONNEL $N^{\circ} 003$ DU 27 MAI 1997 RELATIF A L'ORGANISATION ET A L'EXERCICE DU POUVOIR EN RÉPUBLIQUE DÉMOCRATIQUE DU CONGO
}

\begin{abstract}
En signant, le 27 mai 1997, le Décret-loi constitutionnel $n^{\circ} 003$ relatif à l'organisation et à l'exercice du pouvoir en République Démocratique du Congo, le Chef de l'Etat instaurait certainement un régime constitutionnel intérimaire de très courte durée, en même temps qu'il s' efforçait d'organiser tant soit peu son pouvoir, et répondait aux critiques de l'opinion qui l'accusait de se référer aux statuts de l'AFDL pour nommer les membres du Gouvernement, comme s'il voulait confondre sa formation politique avec l'Etat.
\end{abstract}

En effet, dès son article premier, le Décret-loi constitutionnel précise bien que l'organisation et l'exercice du pouvoir qu'il prévoit ne valent que jusqu'à l'adoption de la Constitution de la Transition par l'Assemblée Constituante; Il se conformait de la sorte à la déclaration de prise du pouvoir qui prévoyait déjà une telle Constitution à breve échéance. Est-ce peut-être pour cela que le Décret-loi sera sommaire, alors que généralement, une Constitution est plus complète? 
En toute hypothèse, le programme politique et constitutionnel annoncé, le 29 mai 1997, par le Chef de l'Etat dans son discours d'investiture, va fausser toute la philosophie qui sous-tend le Décret-loi ${ }^{\circ} 003$ dans la mesure où non seulement la mise en place de la constituante est renvoyée en juin 1998, mais aussi il n'est plus question que de la Constitution de la IIIeme République et non de celle de la Transition.

Quoiqu'il en soit, tout en garantissant l'exercice des droits et libertés individuels et collectifs, le nouveau texte constitutionnel dispose en son article 3 que les institutions de la République sont au nombre de trois, à savoir: le Président de la République, le Gouvernement et les Cours et Tribunaux.

Le pouvoir législatif n'est pas autonomisé par la création d'une institution propre, son exercice est confié au Président de la République qui est, par ailleurs, Chef de l'Exécutif: A ce titre, il légifère par décrets-loi délibérés en Conseil des Ministres, et exerce le pouvoir réglementaire par voie des décrets. Le Chef de l'Etat devient alors le centre d'impulsion et d'ordonnancement de la vie nationale.

Quant au gouvernement, il remplit les fonctions traditionnelles de l'Exécutif dont l'exécution des lois et des décrets présidentiels, la négociation des accords internationaux et la gestion courante des affaires nationales. Ses membres sont nommés discrétionnairement par le Chef de l'Etat devant qui ils répondent de leur gestion. Est-ce pour confirmer cette soumission quils prêteront plus tard le serment de fidélité au Président de la République? Encore que, dans ce cas, il eut fallu organiser préalablement ce serment par un texte juridique pour qu'il soit fondé.

Le pouvoir judiciaire formé par les Cours et Tribunaux a été reconnu comme la seule autorité ayant la mission de dire le droit (articles 11 et 12).

La Cour Suprême de Justice était ainsi mise à l'aise pour recevoir, le 29 mai 1997, le serment constitutionnel du nouveau Chef de l'Etat.

Cela d'autant plus que les magistrats ne sont soumis, dans l'exercice de leurs fonctions, qu'à l'autorité de la loi. L'indépendance de la justice ayant été en principe garantie de ce fait, il reste cependant à la rendre effective pour jeter les bases d'un véritable Etat de droit.

Pour éviter les conflits des lois dans le temps, le Décret-loi $n^{\circ} 003$ abroge, en son article 14 , les textes constitutionnels et réglementaires lui sont contraires. 
Si l'ordre institutionnel ci-dessus décrit a l'avantage de mettre fin au débat constitutionnel ou plus exactement, de le différer momentanément, il engendre néanmoins certaines conséquences dont deux sont parmi les plus importantes.

D'abord, l'article 14 du Décret-loi du 27 mai 1997 abroge les décrets-loi portant nomination de certains membres du Gouvernement, car contraires à la lettre de l'article 5 du nouveau texte constitutionnel.

Dès lors, le mandat des Ministres concernés a été invalidé. Il aurait fallu, pour éviter ce désagrément, les réinvestir par un décret pris par le Chef de l'Etat en vertu de son pouvoir réglementaire.

Loin de relever d'un juridisme creux, cette objection se situe au coeur même des exigences d'un Etat de droit. C'est donc une question de principe et de cohérence.

Ensuite, à y regarder de près, on s'aperçoit que le régime politique établi par le Décret-loi constitutionnel du 27 mai 1997, a tout l'air du présidentialisme.

En effet, le présidentialisme est une déformation du régime présidentiel traditionnel qui consiste à hypertrophier l'Exécutif au détriment du Législatif et à conférer une hégémonie indiscutable au Président de la République. ${ }^{7}$

Cette tendance a d'autant plus de chance de s'accentuer au Congo que non seulement le Parlement est tout simplement inexistant, mais aussi aucun contrepoids politique ou juridictionnel ne s'exerce sur le Chef de l'Etat qui concentre, par ailleurs, tout le pouvoir politique entre ses mains.

L'appréhension est corroborée d'une part, par la suspension des activités des organisations politiques et les faiblesses internes de celles-ci, et d'autre part, par le fait que le Décret-loi Constitutionnel n'offre aucune possibilité de mettre en cause les membres de l'Exécutif:

Bien plus, en l'absence des textes particuliers complétant le Décret-loi, notamment ceux relatifs à l'organisation du Gouvernement, de l'Armée et des autres grands services de l'Etat, le Président de la République est à la fois l'inspirateur et l'animateur de l'action gouvernementale. Quand on sait que son ancienneté dans l'engagement et la lutte politique aux côtés des nationalistes congolais depuis 1960, confère au Président Kabila un charisme incontestable au

\footnotetext{
${ }^{7}$ Pour le présidentialisme, voir notamment : B. JEANNEAU, Droit Constitutionnel et Institutions Politiques, Paris, DALLOZ, 2eme édition, p. 75;
} 
sein de l'AFDL, qu'est à ce jour le véritable siège du pouvoir, on mesure à quel point l'influence du Chef de l'Etat est décisive dans la conduite des affaires nationales.

Le nouveau pouvoir tend alors à s'incarner, aux yeux de l'opinion, en la personne du Président Kabila. Dans ce contexte, le risque d'une dérive autocratique n'est pas à exclure totalement.

Le régime présidentiel du type américain qui semble emporter les faveurs de l'AFDL, serait dévoyé, car, il n'y aurait ni équilibre ni séparation des pouvoirs.

D'où les critiques, parfois acerbes, articulées contre le Président de la République accusé d'être un "Self-made King" et de s'être attribué tous les pouvoirs, y compris celui de Constituant.

Pour fondées que puissent être ces critiques, elles n'empêchent d'interroger l'histoire des Nations. Celle-ci indique que la tabula rasa est aussi classique qu'inhérente au processus de destructuration-restructuration qui suit généralement la prise du pouvoir en dehors du cadre constitutionnel en vigueur.

Lorsque la lutte sur un régime aboutit à son renversement par une révolution ou par les armes (coup d'Etat, guerre de libération etc...), l'on n'enferme pas le nouveau pouvoir dans le carcan juridique antérieur. Il s'organise sur des bases nouvelles et secrète toujours sa propre légalité. Aussi, devient-il le pouvoir constituant originaire qui, à la différence de celui dérivé ou institué par l'ancien édifice constitutionnel, est inconditionné et s'exerce dans le contexte d'une rupture de légalité. La vague des coups d'Etat et des révolutions qui avait soufflé dans le tiers-monde à partir de la seconde moitié des années 60 ainsi que les bouleversements qui ont fait basculer le bloc communiste ces dix dernières années, ont obéi à cette loi de l'histoire.

Par ailleurs, le pouvoir a horreur du vide et le pays ne peut rester indéfiniment en jachère. Apres le départ du Président Mobutu et l'échec des institutions gouvernementales issues de l'Acte constitutionnel de la Transition, il fallait sans tarder pourvoir à la vacance et assurer la continuité de l'Etat, au risque de laisser le pays à la merci de n'importe quel aventurier.

A cet effet, il était illusoire de penser que les vainqueurs laisseraient la place à d'autres forces politiques ou que l'AFDL désignerait à la Présidence de la République quelqu'un d'autre que son leader, Monsieur Laurent-Désiré Kabila. 
C'est dans ce sens que l'on peut comprendre la déclaration de prise de pouvoir du 17 ma 1997 et le Décret-loi constitutionnel ${ }^{\circ} 003$ du 27 mai 1997.

Au demeurant, l'histoire récente de la République Démocratique du Congo ne témoigne-t-elle pas l'existence de quelques précédents en la matière?

En effet, par son Décret-loi Constitutionnel du 29 septembre 1960, le Président Kasavubu, en violation de la loi fondamentale, légitima le Collège des Commissaires Généraux et lui confia cumulativement les pouvoirs exécutif et législatif en attendant l'organisation d'une Table Ronde qui regrouperait tous les leaders politiques en vue de la réconciliation nationale. ${ }^{8}$

En 1965, alors que la Proclamation du Haut-Commandement de l'Armée Nationale Congolaise du 24 novembre 1965 maintenait au point 4 les institutions démocratiques du Congo telles qu'elles étaient prévues par la Constitution $\mathrm{du}$ ler août 1964, le Président Mobutu, en violation de cette Constitution et par la mise en veilleuse de fait du Parlement, s'attribua progressivement le pouvoir législatif par les ordonnances-loi $n^{\circ} 7$ du 30 novembre 1965, 66/92 bis du 07 mars 1966 et 66/621 du 21 octobre $1966 .^{9}$

En France, pour citer un cas de droit comparé, lorsque le général De Gaulle arrive au pouvoir en mai 1958, il obtiendra que soient remis à son Gouvernement non seulement le pouvoir législatif mais aussi le pouvoir constituant, par dérogation, pour ne pas dire en violation de la Constitution de 1946 encore en vigueur, aux fins de "redresser la Nation" française en péril. De Gaulle exercera ainsi les pleins pourvoirs de juin 1958 à février 1959, le temps de mettre en place les institutions prévues par la nouvelle constitution d'octobre 1958 .

Cependant, si en vertu de l'adage salus populi suprema lex, le salut du peuple est la Loi Suprême, le droit de mettre en oeuvre une nouvelle légalité est reconnu à tout nouveau pouvoir généré en dehors de tout ordre constitutionnel, il reste que l'adhésion populaire sur laquelle repose toute légitimité en démocratie n'est acquise que si ce pouvoir répond aux aspirations profondes des citoyens et s'exerce en conformité avec les valeurs ainsi que les normes de la collectivité. ${ }^{10}$

\footnotetext{
${ }^{8}$ NKULU KILOMBO, Plaidoyer pour une transition démocratique au Zaïre, Editions BOPOL, Kinshasa, 1993. IYELEZA, MOYU-MBEY, MASIKA KATSUVA, ISENGINGO \& KAMBERE-ng'ISE, Recueil des textes constitutionnels de la République du Zaïre du 19 mai 1960 au 28 avril 1991, Editions ISE-CONSULT, Kinshasa Avril 1991, page 27.

${ }^{9}$ IYELEZA, MOYU-MBEY op. cit., page 81.

${ }^{10}$ BUFFELAN, J.P., Introduction à la Sociologie politique, Paris, Masson, 1969, page 20.
} 


\section{CHAPITRE II : LEGITIMITE DU GOUVERNEMENT AFDL}

Selon J.P. Buffelan, la légitimité du pouvoir n'est autre chose que le fait qu'il est reconnu comme tel par les membres de la collectivité, ou du moins par la plupart d'entre eux. Un pouvoir est légitime s'il y a consensus à son égard ... La légitimité se fonde donc sur l'adhésion d'une large majorité des citoyens, renforcée par le caractère légal de la passation des pouvoirs et par la reconnaissance accordée par les Etats étrangers. ${ }^{11}$

D'après Le Lexique de termes juridiques, cette légitimité résulte de la conformité du pouvoir aux aspirations des gouvernés notamment sur son origine, sa forme et ses objectifs; ce qui lui vaut l'assentiment général et l'obéissance spontanée. ${ }^{12}$

En toute hypothèse, le pouvoir légitime est approuvé au moins tacitement par la grande majorité des citoyens.

Cependant, la légitimité n'est pas immuable. Elle est soumise aux contingences du temps et dépend de la capacité d'un pouvoir à s'ajuster aux fluctuations de la demande sociale.

Des lors, analyser la légitimité du Gouvernement de l'AFDL, aussi bien au sens de pouvoir exécutif qu'à celui de pouvoir politique tout court, c'est d'abord établir l'existence de l'adhésion populaire à ce gouvernement et sa reconnaissance internationale, tant il est vrai que depuis le vent de la démocratisation, la légitimation externe devient de plus en plus un des critères de viabilité des régimes.

C'est ensuite chercher les fondements de cette légitimité de façon à jauger sa solidité.

A ce sujet, force est de reconnaître que si la légitimité du nouveau pouvoir n'est pas formelle ou n'a pas été formalisée, elle ne demeure pas moins réelle.

\footnotetext{
${ }^{11}$ Ibidem, page 48.

${ }^{12}$ GUILLIEN, R. et VINCENT, J., Lexique de termes juridiques, DALLOZ, Paris, Seme édition, page 254
} 
En effet, l'accueil réservé par la population congolaise à l'AFDL partout ou elle est passée et l'acceptation par tous de Laurent-Désiré Kabila comme Chef de l'Etat avec lequel, au demeurant, tout le monde cherche manifestement à collaborer, confirment l'adhésion du Peuple congolais à leur avènement aux commandes de l'Etat.

De même, la reconnaissance quasi unanime dont le nouveau régime a bénéficié, des le lendemain de sa victoire militaire, de la part des partenaires extérieurs, l'a légitimé sur le plan international.

Toutefois, cette légitimité s'enracine d'abord sur l'histoire du Congo Indépendant d'où elle émerge au fil du temps.

Dans ce sens, il convient de relever que la légitimité de la première législature de la Première République est demeurée d'autant plus vivace, dans la mémoire collective des Congolais, qu'elle avait été cassée tôt et tragiquement par la révocation, suivie de la mort du Premier Ministre Lumumba. Depuis lors, l'ombre de celui-ci n'a cessé de hanter, comme un spectre lancinant, le paysage politique congolais au point que toutes les "révolutions " qui ont émaillé la vie nationale se sont réclamé de lui. C'est le cas de la République Populaire $d u$ Congo proclamée à Stanleyville, de l'insurrection muléliste de 1964 et du Coup d'Etat de l'Armée Nationale Congolaise qui a porté le Général Mobutu au pouvoir, lesquels ont tous cherché à puiser leur propre légitimité dans celle du héros national.

Le même phénomène s'observe avec l'AFDL qui, politiquement, s'identifie volontiers à Patrice-Eméry Lumumba, considéré par tous comme le père du nationalisme congolais et incarnation de cette légitimité démocratique étouffée.

Aussi a-t-elle clairement situé, dans sa Déclaration de prise du pouvoir du 17 mai 1997, le début de la guerre de libération au 14 septembre 1960, date du premier coup d'Etat de Mobutu contre les institutions démocratiquement élues. C'est sans doute cet attachement à la légitimité de l'Indépendance qui a conduit l'Alliance à réhabiliter les symboles de la Première République dont le drapeau, l'hymne national, le nom du pays et l'ancienne unité monétaire.

En vérité, il y a lieu de reconnaitre cependant, que cette légitimité n'a pu garder son actualité que parce que les gouvernants qui se sont succédé à la direction du pays depuis la révocation de Lumumba, n'ont pas réussi à réaliser la concorde et la prospérité nationales ainsi que la sécurité des personnes et des biens, objectifs ultimes de tout pouvoir. 
A ce sujet, point n'est besoin de nous appesantir dans cette étude sur la crise zaïroise, tant il est vrai qu'une littérature aussi abondante que variée y a été consacrée au pays et à l'étranger.

Il convient, toutefois, de rappeler qu'en ce qui concerne plus particulièrement le règne du Président Mobutu, la contestation estudiantine quasi endémique, les deux guerres du SHABA (1977 et 1978), la fronde parlementaire des années 80, le délabrement du tissu économique national, l'endettement excessif du pays sans contrepartie rentable, l'aggravation de la misère du Peuple et la mise en quarantaine du Zaïre par ses principaux partenaires extérieurs, sont parmi tant de faits qui ont révélé au grand jour la faillite du système de la IIème République.

C'est sur cette toile de fond de méga-crise qu'une opposition interne a pris corps pour atteindre son point culminant à la Conférence Nationale Souveraine, C.N.S.

Ainsi que l'affirme Maurice Kamto dans "l'urgence de la pensée", le fondement de celle-ci est à trouver dans la rupture de légitimité qui a affecté à la fois le pouvoir d'Etat, les Institutions de la République incapables de répondre à la demande sociale, et même le droit en tant qu'instrument de régulation des rapports sociaux. ${ }^{13}$

A un moment où la communauté internationale lasse des dictatures encombrantes, et le Peuple congolais étaient en quête d'une alternative à Mobutu et à son régime, la CNS est apparue comme une institution de crise ayant pour mission essentielle de réaliser le compromis politique fondateur du nouveau contrat social qui plonge ses racines à la fois dans le regain démocratique mondial et dans la volonté du Peuple de rompre avec l'ancien système des valeurs. A ce titre, elle passait dans l'imaginaire populaire pour la voie royale pour une solution concertée de la crise et susceptible de conduire à un changement politique non violent ni décrété unilatéralement par le Président Mobutu.

C'est pourquoi la CNS a bénéficié d'une très large légitimité de la part du Peuple congolais qui est resté attaché à son projet de société jusqu'à ce jour.

Le processus de changement proposé par la Conférence passait par une transition démocratique qui devait être la préfiguration de la IIIème République. Pendant cette transition, le Haut Conseil de la République, émanation de la CNS, devenait le siège de la légitimité du pouvoir dont l'Exécutif était confié à un Premier

\footnotetext{
${ }^{13}$ KAMTO Maurice, L'Urgence de la pensée, Yaoundé, Editions Mandare, 1994, p. 185.
} 
Ministre élu par cette Conférence.

Cependant, en raison du soutien de l'Armée au Président Mobutu, le rapport des forces sur le terrain est demeuré en sa faveur.

Ainsi, quoiqu'ayant affaibli considérablement le régime Mobutu avec l'aide de l'opposition non armée et du Peuple lui-même, le schéma de la CNS n'a pas réussi à chasser le dictateur du pouvoir. Bien au contraire, "dribblant" tout le temps la classe politique, le Président Mobutu a bloqué la transition, le plus souvent par les armes, et s'est mis, par dessus tout, à planter le décor de la reproduction organique de son système, même au-delà de sa propre mort. Comme en 1960 avec la première législature, la légitimité de la CNS était en train d'être tournée en dérision par la même armée au service de la même personne. La méga-crise s'est aggravée, plongeant davantage le Peuple dans le désespoir.

C'est dans ce contexte qu'en septembre 1996, la guerre des BANYAMULENGE a éclaté au Sud-Kivu comme la goutte d'eau qui a fait déborder le vase d'une crise généralisée dont les congolais supportaient de plus en plus mal le poids.

Bénéficiant d'un appui important en matériel et en hommes de troupes de la part du Rwanda, de l'Ouganda, du Burundi, de l'Angola et de la Zambie qui étaient exaspérés par la présence d'un voisin aussi nuisible que le Zaïre de Mobutu, cette guerre, au départ limitée aux objectifs de défense idemnitaire s'est vite muée, sous la conduite de l'AFDL, en une lutte de libération du Congo avec la bénédiction de certains pays occidentaux.

Le terrain étant favorable car préparé par l'opposition interne non armée, la guerre va s'étendre rapidement comme un feu de brousse pour atteindre Kinshasa en sept mois seulement, malgré la vaste étendue du territoire national.

Ainsi, grâce aux mêmes moyens et soutien extérieur qui ont permis au Président Mobutu d'accéder au pouvoir et de s y maintenir 32 ans durant, l'AFDL a réalisé le rêve des congolais en les débarrassant de l'ancien Chef de l'Etat

Il n'est que normal dans ces conditions que le Peuple l'accueille en libérateur. Au surplus, à en juger par le programme minimum consigné dans ses statuts et par certains actes de son gouvernement, l'Alliance s'appuie aussi de façon à peine voilée sur la légitimité issue de la CNS en dépit des déclarations de son Président qui la rejette. ${ }^{14}$

${ }^{14}$ Journal le SOFT, op. cit. 
Néanmoins, cette légitimité demeure celle de la résignation et de la révolte contre un homme et le système qu'il a incarné longtemps.

Elle est par conséquent fragile puisque'elle repose sur un référentiel qui s'effrite avec la fin du règne du Président Mobutu. Celui-ci parti, en effet, la méga-crise revient à la surface avec son cortège des revendications socio-politiques que le seul changement d'hommes à tête du pays ne suffit pas pour apaiser. D'où la nécessité de poursuivre le processus de légitimation en ouvrant des perspectives nouvelles qui transcendent la logique de la guerre car, si les armes ont été indispensables pour faire tomber la dictature, il n'est pas évident qu'elles sont nécessairement le moyen approprié pour convaincre le Peuple.

\section{CONCLUSION}

L'analyse de l'ordre institutionnel de la nouvelle transition et de la légitimité du gouvernement de l'AFDL peut conduire aux constatations ci-après.

1. Le Décret-loi constitutionnel $n^{\circ} 003$ du 27 mai 1997, matrice de l'architecture institutionnelle actuelle, est de portée limitée aussi bien dans le temps qu'en rapport avec les matières qu'il régit.

En effet, normalement la Constituante annoncée par la Déclaration de prise de pouvoir du 17 mai 1997 aurait dû déjà doter le pays d'une nouvelle Constitution de la transition.

Par ailleurs, après l'abrogation de la Loi $n^{\circ} 82 / 006$ du 27 fevrier 1982 portant organisation territoriale, politique et administrative de la République par celle votée par le Haut Conseil de la République-Parlement de Transition, elle-même tombée en désuétude, l'organisation de la territoriale en République Démocratique du Congo ne repose sur aucune base juridique.

On ne connaît ni la nature, ni la dénomination, ni les organes, et encore moins les ressources de nouvelles entités retenues parle nouveau pouvoir. Celles-ci fonctionnent tant bien que mal selon le savoir-faire de leurs gestionnaires 
respectifs.

De même, en l'absence des textes juridiques précis, on ignore la structure de l'armée, des services de l'ordre et de sécurité. Les rapports entre ces grands corps de l'Etat ne sont pas définis.

Le Décret-loi constitutionnel est peu détaillé sur l'organisation et le fonctionnement de l'Exécutif qui gère le pays sans contrôle politique ni juridictionnel institué.

La structuration du pouvoir et de l'appareil de l'Etat est donc inachevée.

C'est pourquoi, il est impérieux d'asseoir la République Démocratique du Congo sur une véritable constitution républicaine qui est la marque des Etats modernes et un des facteurs de développement politique des peuples.

2. Le cadre institutionnel établi par la Déclaration de prise de pouvoir et le Décret-loi constitutionnel favorise le Président de la République, et risque, si l'on n'y prend garde, d'instaurer un régime à souveraineté présidentielle.

Dans ce type de régime, le Président de la République est l'unique et la véritable source du pouvoir. C'est l'homme de qui émane l'autorité et qui seul en délègue l'exercice aux personnes de son choix. ${ }^{15}$

On retrouve là la tendance normale du pouvoir en République Démocratique du Congo depuis 1960. Hormis l'étape de la CNS, toutes les variations subies par le régime politique congolais suivent une seule direction. le renforcement des pouvoirs présidentiels. La trajectoire vers le présidentialisme est donc restée ascendante.

3. La Déclaration de prise de pouvoir du 17 mai 1997, le Décret-loi constitutionnel du 27 mai 1997 et la manière dont le pouvoir s'exerce dans la réalité créent un environnement propice au monolithisme.

Toutefois, il est peu probable que cette orientation rencontre un consensus social favorable à sa consolidation. En effet, la fin de l'empire soviétique, l'écroulement du Mur de Berlin, la chute de Ceaucescu et le phénomène Mandela, médiatisés tous à qutrance par la presse internationale, ont laissé une empreinte indélébile dans le mental des peuples africains qui ne sont plus disposés à accepter tout ce

${ }^{15}$ LUTUNDUA APALA, C ., op. cit. p 147. 
qui prête à la dictature. Le mythe des guides éclairés, des pères de la Nation des timoniers etc... est tombé. Il n'est plus qu'une coquille vide.

Par ailleurs, on peut se demander dans quelle mesure le peuple congolais dont la culture et la conscience politiques se sont aiguisées à la faveur de l'apprentissage démocratique de sept dernières années, pourra s'accommoder longtemps d'un monolithisme que certains ont fait vite, du reste, d'assimiler, à tort ou à raison, aux pratiques du Parti-Etat.

Dès lors, il est à craindre qu'à terme le durcissement du pouvoir et un blocage étanche de l'expression plurielle de la pensée politique ne génèrent des frustrations susceptibles de déstabiliser le pays.

Le défi à reveler dans ce contexte devient celui d'organiser le pluralisme politique au Congo de manière à le rationaliser et à en faire un instrument efficace du développement.

C'est pourquoi, il est indispensable de penser d'ores et déjà à définir le nouveau cadre d'exercice des activités politiques et sociales en République Démocratique du Congo. Aussi devra-t-on procéder entre autres à la révision des statuts de l'AFDL qui ne peut plus se définir comme une organisation politico-militaire, ainsi que le stipulent le préambule et l'article 12 de ses statuts.

En effet, la défense nationale et la sécurité du territoire étant une des prérogatives régaliennes de l'Etat dans le cadre de sa souveraineté, il est inconcevable qu'une organisation politique, même celle au pouvoir, puisse disposer d'une armée.

De même, la conclusion qui est faite à l'article 23 des dits statuts entre le patrimoine commun de la Nation et celui de l'AFDL n'est pas conforme aux exigences d'un Etat démocratique.

Le nouveau Congo doit éviter de tomber dans le piège de la mode selon laquelle, dans le contexte africain, la bonne gouvernance l'emporterait sur la démocratie, comme si l'une était incompatible avec l'autre.

En vertu de cette bonne gouvernance, la dictature s'appellerait le régime fort; aux dictateurs d'hier succéderaient des leaders charismatiques; le respect des droits de l'homme céderait la place aux performances économiques, qui ont pour noms, maîtrise de l'inflation, stabilisation de la monnaie, déficit budgétaire faible ou nul, balance commerciale excédentaire ou du moins équilibrée, paiement de la dette extérieure pour ne pas dire transfert des capitaux vers l'Occident et taux de 
croissance positif du PIB; la prospérité de l'économie créerait spontanément des espaces de liberté dont les africains n'ont pas, du reste, tellement besoin pour le moment et la démocratie, on verra plus tard!

Une telle conception de la bonne gouvernance nous parait étriquée et dangereuse, car liberticide.

La bonne gouvernance est une philosophie globale de gestion du destin d'une Nation impliquant tout autant le bien-être économique et social que le développement politique de nos peuples.

4. La légitimité du nouveau pouvoir ne peut entrer dans la typologie classique, puisqu'elle ne relève ni de la tradition, ni du charisme personnel du Chef de l'Etat et encore moins de l'ancienne légalité. Néanmoins, elle est réelle, populaire et consensuelle, mais fragile.

C'est pourquoi, il est indispensable de poursuivre la légitimation du pouvoir par la mise en oeuvre du programme politique annoncé par le Président de la République. Pour être salutaire, ce programme devra offrir un cadre approprié à la réconciliation nationale. Celle-ci n'est pas synonyme de formation d'un Gouvernement d'union nationale. Autrement, elle devient un horizon qui s'éloigne chaque fois qu'on s'y approche.

La réconciliation est avant tout l'accord sur le nouveau contrat social qui régira désormais le destin de tous les congolais. Ce contrat constitue le fond commun minimal qui assure la stabilité de toute société.

En l'espèce, il s'agira dans le cadre d'une Constituante ou d'une Commission Constitutionnelle, d'accorder les violons entre les options de la Conférence Nationale Souveraine et celles de l'AFDL, notamment en ce qui concerne:

- la réforme de l'Etat, en ce compris l'organisation et l'exercice du pouvoir ainsi que celui des libertés et droits fondamentaux;

- l'établissement de l'Etat de droit;

- la poursuite du processus de démocratisation et l'organisation des élections;

- l'instauration de la Justice sociale et de la solidarité;

- les rapports entre les différentes composantes de la Nation et du pays. 
C'est dans ces conditions qu'il sera possible de donner toutes ses chances de réussite au programme de reconstruction nationale initié par le Gouvernement, réussite dont dépend également la légitimité du pouvoir.

Les Congolais auront alors compris le message d'un grand sage africain, en l'occurrence l'ancien Président tanzanien Julius Nyerere, qui disait récemment qu'il $\mathrm{n}$ y a pas de reconstruction sans réconciliation nationale.

Kinshasa, le 2 septembre 1997 


\section{BIBLIOGRAPHIE}

AFDL, Statuts, in Journal Le Soft du 30 mai 1997.

BUFFELAN, J.P., Introduction à la sociologie politique, Masson, Paris, 1969

DUVERGER, Maurice, Institutions politiques et droit constitutionnel, Thémis, PUF, t. I, 18e éd., 1990.

GOOSSENS, P. CH. et ZWETKOFF, C., Notes inédites de cours des institutions politiques comparées, Université de Liège, s.d,

GUILLIEN, R. et VINCENT, J., Lexique de termes juridiques, DALLOZ, Se éd., Paris.

IYELEZA, MOYU-MBY, MASIKA-KATSUVA, ISENGINGO \& KAMBERE ng'ISE, Recueil des textes constitutionnels de la République du Zaïre du 19 mai 1960 au 28 avril 1991, Ed. ISE-CONSULT, Kinshasa, avril 1991.

JEANNEAU, B., Droit constitutionnel et institutions politiques, DALLOZ, 2e éd;, Paris.

KAMTO, Maurice, L'urgence de la pensée, Mandare, Yaoundé, 1994.

LUTUNDULA APLA, Christophe, Exécutif du type présidentiel: évolution politique et constitutionnelle nu Zaïre, Mémoire de Licence, Faculté de Droit, UNAZA, Kinshasa, 1977.

NKULU KILOMBO, Plaidoyer pour une transition démocratique au Zaïre, Ed. BOPOL, Kinshasa. 